\title{
The Study of Knowledge Support Mechanism for Valve Product Design
}

\author{
Lanzhi $\mathrm{Gu}^{1, \mathrm{a}^{*}}$, Jiangyan $\mathrm{Jin}^{2, \mathrm{~b}}$, Yixiang Fang ${ }^{2, \mathrm{c}}$ \\ ${ }^{1}$ Hebei Chemical and Pharmaceutical College, Shijiazhuang 050026, China \\ ${ }^{2}$ College of Mechanical\&Electronic Engineering, Hebei University of Science and Technology, \\ 050018, Shijiazhuang, China \\ agulanzhi1971@126.com, bjinjy1108@163.com, ‘fang_yixiang@163.com
}

Key Words: Valve product design; Knowledge support mechanism; Knowledge base; Product design process; CAD system based on knowledge

Abstract. By the analysis of the knowledge constitution in the field of valve product design, combined with the CAD system based on knowledge , the knowledge support model is constructed, in which the knowledge support mechanism facing product design process is discussed, the knowledge support mode of each design stage is clarified. In addition, the constitution and constructing mode of knowledge base is decided, which will lay the foundation for the detailing and realizing of knowledge base.

\section{Introduction}

As a kind of general fluid machinery, valves are widely used in petroleum, chemical industry, metallurgy, electric power, long distance pipeline and other industrial fields. There are many kinds of walves in different fields. Valve products have high degree of serialization. Lots of standard parts and interchangeable parts are used in valves. There are a lot of professional knowledge and design calculation model about valve product design, which is specific, regularity and can be followed. To realise the reuse of domain knowledge in a valve CAD system based on knowledge[1], we induced the knowledge constitution in the field of valve product design, analyzed the valve product design process, established the knowledge support mechanism, built the knowledge base.By the realization of knowledge reuse, the labor intensity of designer can be greatly reduced, the design efficiency and quality can be improved.

\section{Constitution of valve product design domain knowledge}

As a general mechanical product, the valve design has the characteristics of general mechanical products design. At the same time, valve design must follow the unique design specification of valve industy and production enterprises. The valve design knowledge include the relevant industry standards , design criteria, design experience design cases and so on. To realize the knowledge support for valve product design, we must analysis the constitution of valve product design domain knowledge in the first place. Based on deep study on the product structure and product design process of the related enterprise, we classified the valve product design domain knowledge into three aspects: design standard, design experience and design case[2,3].

Standard knowledge. The valve product design should be carried out under the relevant standard ang specification. Valve product design standards can be divided into international standards, national standards, industry standards and enterprise standards. In addition, these standars are abundant and complex. Different standards respectively have their own scope of application. Some 
are applicable to all kinds of valve products, such as the standard series about nominal diameter and nominal pressure. Some are for specific valves varieties, such as butterfly valve seat minimum flow channel size criteria, length of flange connection structure of butterfly valve series, etc. In order to realize the effctive knowledge support for specific design system, design standards should be seleccted,summarized and classified before representation and storage,

Experience knowledge. A lot of experience knowledge will be accumulated in the long-term process of product design by desigers. These empirical knowledge mainly reflects in the value of some of the key design parameters, empirical design algorithm, typical structure of specific products and the supply of outsourcing parts information, etc. Some empirical knowledge exists in designer's mind, some is presented in the finished product design instance, which is often not focus and clear enough. Design experience, however, as an important part of the design domain knowledge, is the precious resources for enterprises and play an important role in product development. It is the premise and foundation of knowledge support to dig, settle and regularize experience knowledge.

Case knowledge. Product cases are the ultimate sign of design results, which can be embodied in $2 \mathrm{~d}$ drawings, $3 \mathrm{~d}$ models and so on. A product case involves its parts structure composition and the design data of whole design process[4,5]. Product cases include successful and failure design experience, reflect the use of design principles, design criteria and empirical knowledge. By studying different design cases of the same kind of valve products, we can identify the structure of the specific valve and its components generating order, and induce the different typical structure of any part. The design process data of a product case can support the reuse and modifying of this case, and provide experience for the design of new products. Therefore, case knowledge is the key and core of the valve design domain knowledge.

\section{The CAD system based on knowledge for valve}

Overall framewok. The CAD system based on knowledge of the valve is constructed on the platform of general 3d CAD software and supported by database management system. The geometric models of valve products are built through parameterized modeling tools of the common software. The application programe is developed in the secondary development environment offered by general CAD software, which navigates the valve product design process with support from knowledge base. The system overall framework is shown in figure 1.

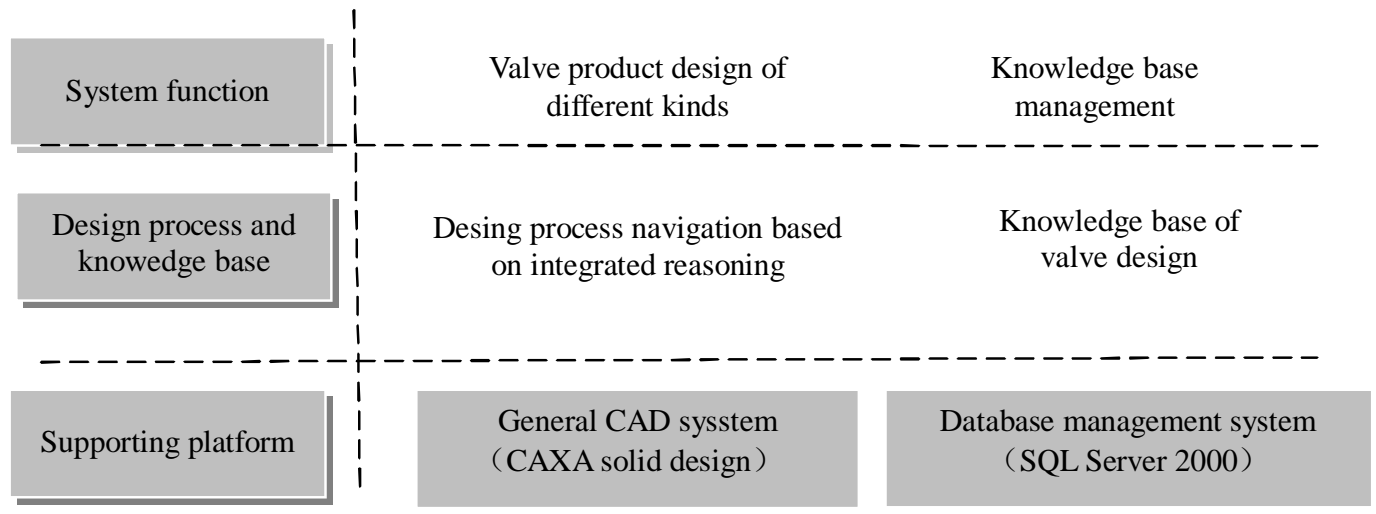

Figure 1. Overall framework of the CAD system based on knowledge for valve

Design process of valve products. The product design process in theCAD system based on knowledge is shown in figure 2 . At the beginning of valve design, according to the use requirements 
and working conditions, overall design demands should be confirmed, which involve nominal pressure, nominal diameter, design temperature ,etc. Then the overall design demands can be used as a retrieval condition for instance retrieval. If there is not any case which can be reused, the major structure design of valve is carried. Aftr the key technical parameters of major stucture is determined, the three-dimension parametric design based on design units will be developed. When the major structure design is completed, the design of a valve product will be finished after the selecting of actuator. The design process will end after the case storage.

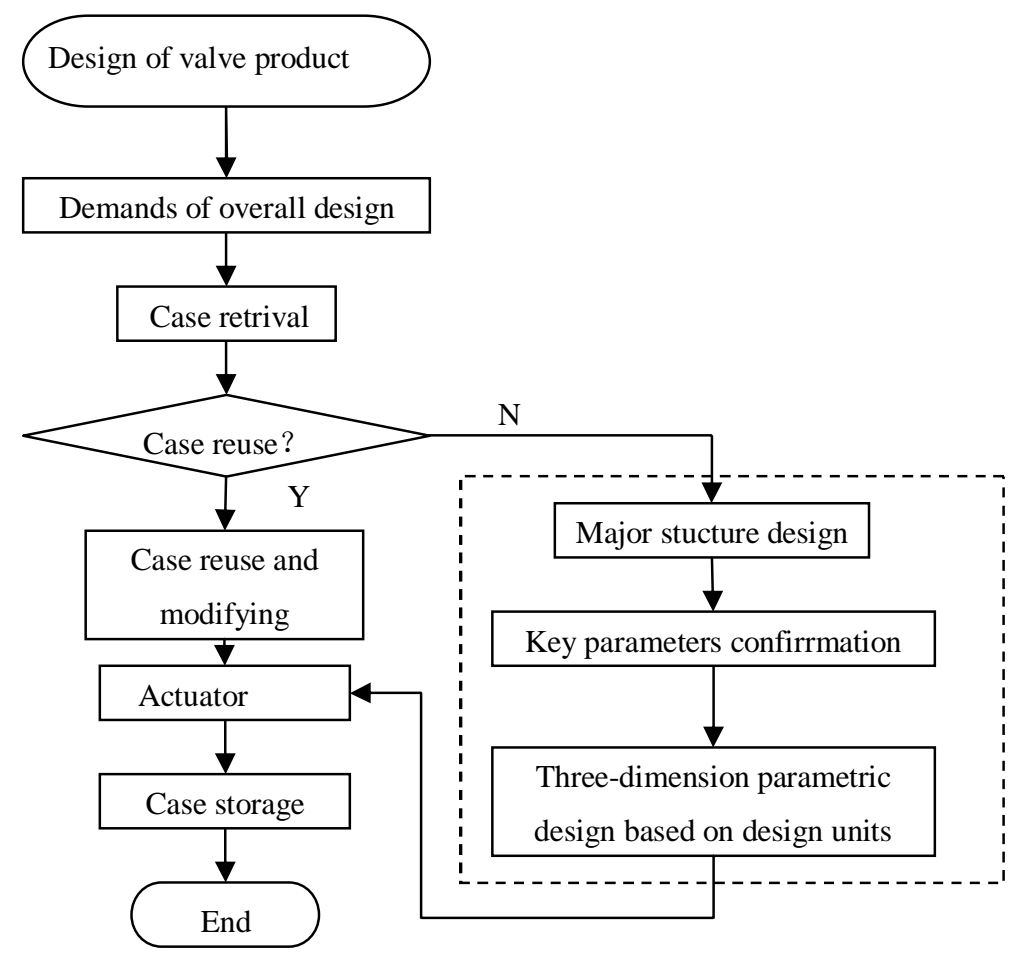

Figure 2. Design process in the CAD system based on knowledge for valve

\section{The knowledge support model}

In the valve $\mathrm{CAD}$ system based on knowledge, the reaearch of knowledge support mechanism is developed on the design process of valve products. After analyzing the knowledge support demand of each node in the design process, the support mechanism can be identified. Due to the strucural difference is big between the different types of valves, such as butterfly valve and the goggle valve, the product design is carried out classifiedly. But different types of valve design follows the basic consistent design process and implementation mechanism, therefore, the knowledge support mode can be unified, which is shown in figure 3 .

\section{Constitution and constructing mode knowledge base}

Through the above research, the knowledge support mechanism in valve producst design process is identified. On this basis, the function of the knowledge base on supporting, sharing and updating is clear. The content of the specific knowledge support for valve design process is confirmed. The building mode of knowledge base is determined.

Constitution of knowledge base. In the model of knowledge support model of product design process, knowledge support includes the relevant design standards, design criteria, experiencee data, and checking algorithm, product case, process data, etc., which almost covers all aspects of the valve product domain knowledge. In addition, the browsing demand for the knowledge should also 
be considered when the base is constructed. There are so many different kinds of knowledge which is hierarchy and complex. A reasonable organization strategy will ensure the validity of the knowledge and be advantageous to the knowledge reuse and updating. With reference to the principle of classification of domain knowledge, the constitution of knowledge base is divided into three modules: design standards, design experience and product cases, which is shown in figure 4.

Constructing mode of knowledge base As the background support for a valve CAD system, in addition to providing intellectual support for the valve product design ,the knowledge base must regularly store the deign process data and new product cases, to ensure the retrieval, reuse, modifing of case, provide guarantee for the subsequent data mining and experience reference. At the same time, a management system can directly manage the knowledge base.The relationship of knowledge base with the management system and valve design process is shown in figure 5.

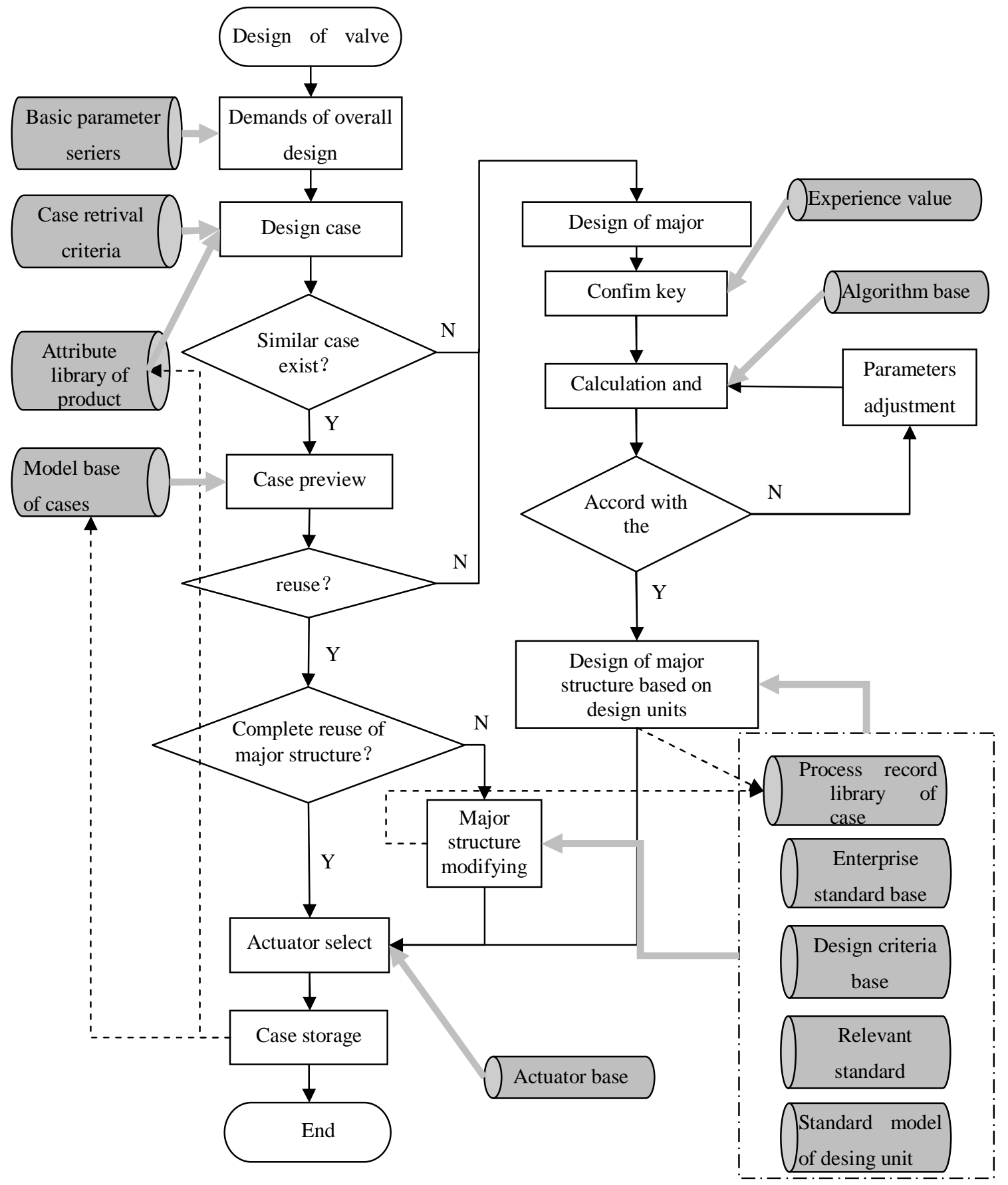

Figure 3. Kowledge support model in design process of valve design 


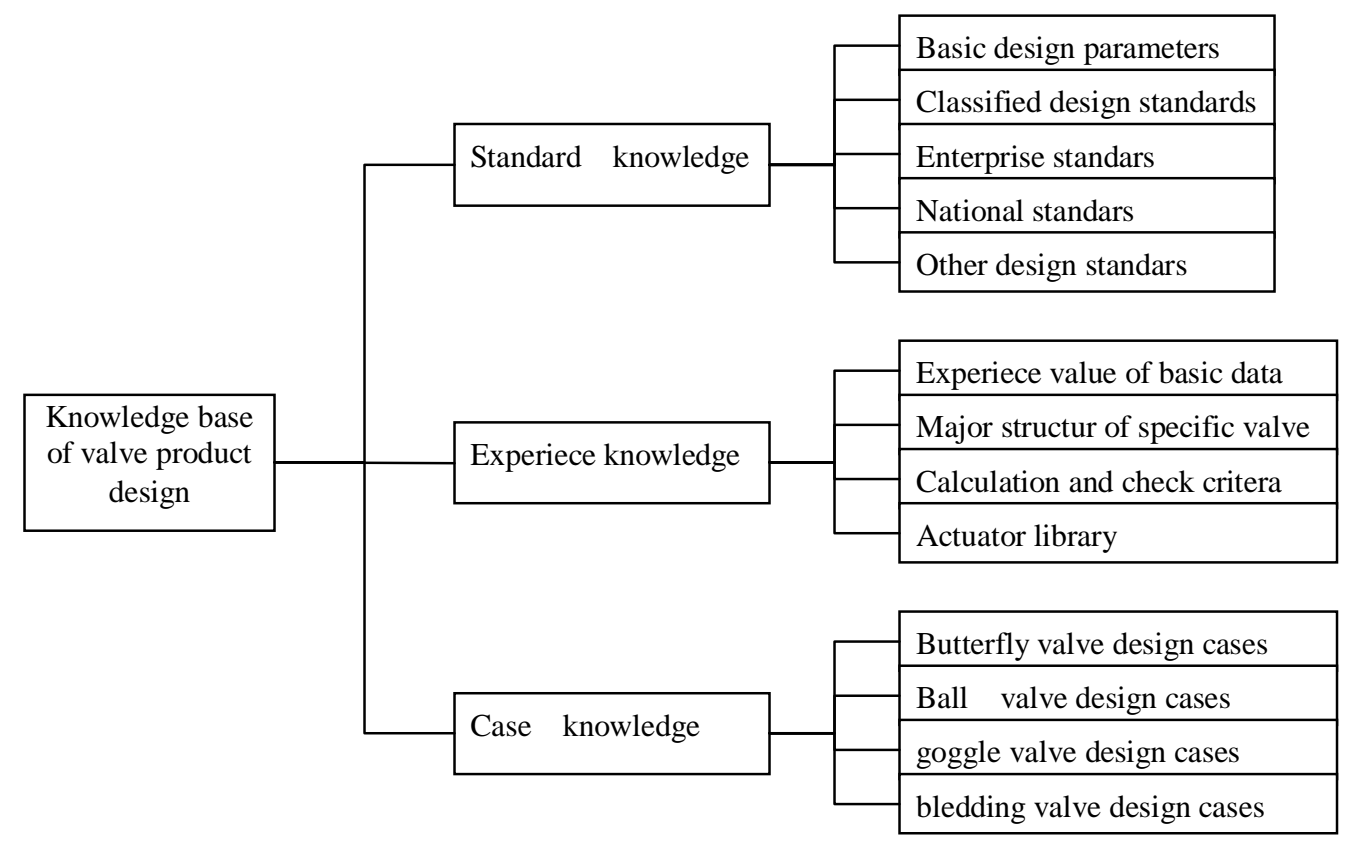

Figure 4. The constitution of knowledge base

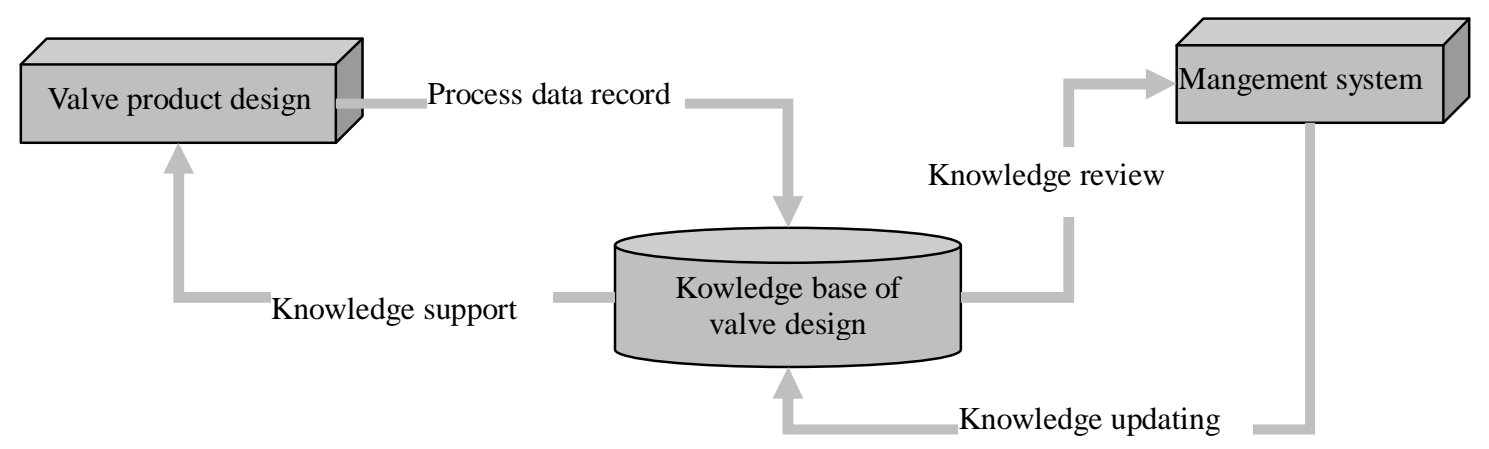

Figure 5. Constructing mode of knwledge base

\section{Conclusion}

In this paper, the design knowledge of valve product is summariesed. Combining with the specific valve CAD system, the knowldege support mechanism is confirmed. The model of knowledge support facing design process is established. The constitution and construcing mode of knowledge base is identified, which lays a foundation for further detailed design and implementation of the knowledge base.

\section{Acknowledgement}

This paper has been sponssored by Youth fund of Hebei province education department (Project No: QN2014207 )

\section{References:}

[1] H.C. Yang, W.H. Liao. 3D CAD Technology an Application of KBE(Chinese). Bejing: Science Press, 2005

[2] A mjad Waheed, Hojjat Adeli. Cased-based Reasoning in Steel bridge Engineering. 
Knowledge-based System, 2005(18):37-46

[3] E. THOMAS, L. THOMAS. Default Reasoning from Conditional Knowledge Bases: Complexity and Tractable Cases. ArtificialIntelligence, 2000, 124(2): 169-241

[4] D. LEAKE, R. SOORJAMURTHI. When Two Case Bases are Better than One: Exploiting Multiple Case Bases. Case-based Reasoning and Development 4th International Conference on Case-based Reasoning. Berlin, Germany: Springer-Verlag, 2001: 321-335

[5] B.J. HICKS, S.J. CULLEY, R.D. ALLEN, et al. A Framework for the Requirements of Capturing, Storing and Reusing Information and Knowledge in Engineering Design. Information Management, 2002, (22): 263-280 